Macmillan

Master

Series

\title{
Work Out
}

\section{Principles of Accounts}

\section{for First Examinations}




\section{The titles \\ in this \\ series}

For First Examinations

Biology

Chemistry

Computer Studies

English Language

Mathematics

Physics

Principles of Accounts

Statistics

For examinations at Advanced Level

Applied Mathematics

Biology

Chemistry

Physics

Pure Mathematics

Statistics

For examinations at college level

Mathematics for Economists

Operational Research 
Macmillan

Master

Series

\section{Work Out}

\section{Principles of Accounts}

for First Examinations

P. Stevens

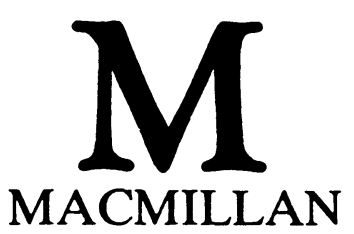


All rights reserved. No reproduction, copy or transmission of this publication may be made without written permission.

No paragraph of this publication may be reproduced, copied or transmitted save with written permission or in accordance with the provisions of the Copyright Act 1956 (as amended).

Any person who does any unauthorised act in relation to this publication may be liable to criminal prosecution and civil claims for damages.

First published 1986

Reprinted (with corrections) 1986

Published by

MACMILLAN EDUCATION LTD

Houndmills, Basingstoke, Hampshire RG21 2XS

and London

Companies and representatives

throughout the world

Typeset by TecSet Ltd,

Wallington, Surrey

British Library Cataloguing in Publication Data

Stevens, Paul

Work out principles of accounts. -

(Macmillan work out series). - (Macmillan master series).

1. Accounting

I. Title

$657 \quad$ HF5635

ISBN 978-0-333-39659-9 ISBN 978-1-349-18141-4 (eBook)

DOI 10.1007/978-1-349-18141-4 


\section{Contents}

Acknowledgements

Introduction

ix

1 Principles of Double-entry Book-keeping

1.1 Introduction

1.2 Types of account

1.3 Rules of double-entry book-keeping

1.4 An illustration of the account

1.5 Balancing off the account

1.6 Purchases and sales returns

1.7 Worked examples

1.8 Further exercises

2 The Trial Balance

2.1 Introduction

2.2 Purpose of the trial balance

2.3 Worked examples

2.4 Further exercises

3 Trading and Profit and Loss Accounts $\quad 16$

$\begin{array}{lll}3.1 & \text { Explanation } & 16\end{array}$

$\begin{array}{lll}3.2 & \text { The trading section } & 16\end{array}$

$\begin{array}{ll}3.3 \text { The profit and loss section } & 17\end{array}$

$\begin{array}{lll}3.4 & \text { Preparation of trading, profit and loss account } & 17\end{array}$

$\begin{array}{ll}3.5 \text { Other trading account expenses } & 18\end{array}$

$\begin{array}{ll}3.6 \text { Worked examples } & 18 \\ 3.7 & \text { Further exercises }\end{array}$

$\begin{array}{lll}3.7 & \text { Further exercises } & 20\end{array}$

4 Balance Sheet $\quad 21$

4.1 Purpose 21

4.2 Preparation of the balance sheet 21

4.3 Balance sheet equation 22

4.4 Capital and revenue expenditure $\quad 23$

$\begin{array}{ll}4.5 \text { Worked examples } & 23\end{array}$

$\begin{array}{lll}4.6 & \text { Further exercises } & 23 \\ \end{array}$

5 Cash Book and Cash Discounts 26

5.1 Cash book 26

$\begin{array}{ll}5.2 \text { Cash discounts } & 27\end{array}$

5.3 Cash discounts in the cash book 27

$\begin{array}{lll}5.4 \text { Petty cash } & 28\end{array}$

5.5 Worked examples $\quad 28$

5.6 Further exercises 34 
6 Bank Reconciliation $\quad 39$

6.1 Introduction 39

6.2 Types of differences 39

6.3 Identifying the differences 40

6.4 Preparing the reconciliation 40

6.5 Worked examples 40

6.6 Further exercises 42

7 Day Books and VAT $\quad 45$

$\begin{array}{lll}7.1 & \text { Introduction } & 45\end{array}$

$\begin{array}{ll}7.2 \text { Cash book } & 45\end{array}$

$\begin{array}{lll}7.3 & \text { Petty-cash book } & 45\end{array}$

$\begin{array}{lll}7.4 & \text { Purchases day book } & 45\end{array}$

$\begin{array}{lll}7.5 & \text { Purchases returns day book } & 46\end{array}$

7.6 Sales day book 46

$\begin{array}{lll}7.7 & \text { Sales returns day book } & 47\end{array}$

$\begin{array}{lll}7.8 & \text { The journal } & 47\end{array}$

$\begin{array}{ll}7.9 \text { Value added tax } & 47\end{array}$

7.10 Worked examples $\quad 48$

7.11 Further exercises 49

8 Errors and Suspense Accounts $\quad 51$

8.1 Errors not affecting the trial balance 51

8.2 Correction of errors 51

8.3 Suspense accounts $\quad 53$

8.4 Worked examples $\quad 53$

8.5 Further exercises $\quad 56$

9 Bad Debts and Bad Debts Provision $\quad 58$

9.1 Writing off bad debts

9.2 Making a provision for future bad debts 58

$\begin{array}{ll}9.3 \text { Adjusting the provision } & 59\end{array}$

$\begin{array}{ll}9.4 \text { Worked examples } & 59\end{array}$

$\begin{array}{lll}9.5 & \text { Further exercises } & 61\end{array}$

10 Control Accounts $\quad 63$

10.1 Introduction 63

10.2 Control accounts and double-entry 63

10.3 Maintaining control accounts $\quad 64$

10.4 Transferring accounts $\quad 64$

$\begin{array}{ll}10.5 & \text { Sectionalisation of ledgers }\end{array}$

$\begin{array}{ll}\text { 10.6 Sales and purchases ledgers } & 65\end{array}$

$\begin{array}{ll}10.7 \text { Worked examples } & 65\end{array}$

$\begin{array}{lll}10.8 & \text { Further exercises } & 68\end{array}$

11 Depreciation and Disposal of Assets $\quad 70$

$\begin{array}{ll}11.1 & \text { Introduction }\end{array}$

$\begin{array}{ll}11.2 \text { Meaning of depreciation } & 70\end{array}$

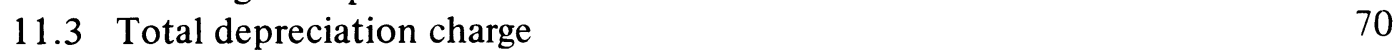

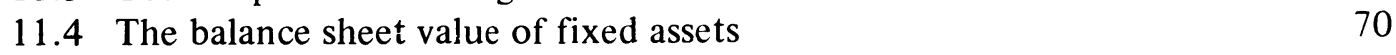

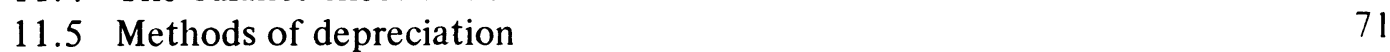

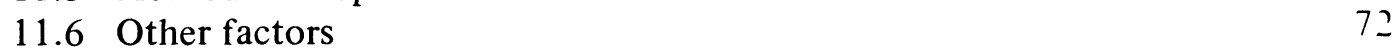

$\begin{array}{ll}11.7 & \text { Accounting entries }\end{array}$

$\begin{array}{lll}11.8 & \text { Fixed asset disposals } & 73\end{array}$

11.9 Assets purchased/sold during year 74

11.10 Worked examples $\quad 74$

11.11 Further exercises $\quad 77$ 
12 Year End Adjustments $\quad 81$

12.1 Introduction 81

12.2 Accrued expenses 81

12.3 Prepayments $\quad 82$

12.4 Sundry stocks $\quad 82$

12.5 Summary $\quad 83$

12.6 Worked examples $\quad 83$

$\begin{array}{lll}12.7 & \text { Further exercises } & 88\end{array}$

13 Departmental Accounts $\quad 93$

13.1 Introduction 93

13.2 Expenses 93

13.3 Preparation of departmental accounts 93

$\begin{array}{ll}13.4 \text { Worked examples } & 94\end{array}$

13.5 Further exercises 98

14 Incomplete Records $\quad 99$

14.1 Introduction 99

14.2 Statement of affairs 99

$\begin{array}{ll}14.3 \text { Trading and profit and loss account from } & 100 \\ \text { incomplete records } & 102\end{array}$

14.4 Goods stolen or lost 102

$\begin{array}{ll}14.5 & \text { Worked examples } \\ 14.6 & 103\end{array}$

$\begin{array}{lll}14.6 & \text { Further exercises } & 108\end{array}$

15 Income and Expenditure Accounts 112

$\begin{array}{lll}15.1 \text { Introduction } & 112\end{array}$

$\begin{array}{ll}15.2 \text { Accumulated fund } & 112 \\ 15.3 \text { Members'subsciptions } & 112\end{array}$

$\begin{array}{ll}15.3 \text { Members' subscriptions } & 112 \\ 15.4 \text { Bar trading account } & 113\end{array}$

$\begin{array}{lll}15.4 & \text { Bar trading account } & 113\end{array}$

$\begin{array}{ll}15.5 \text { Other club activities } & 114\end{array}$

$\begin{array}{lll}15.6 & \text { Receipts and payments account } & 114\end{array}$

15.7 Worked examples 114

15.8 Further exercises 119

16 Partnership Accounts: 1

$\begin{array}{lll}16.1 & \text { Definition } & 123\end{array}$

$\begin{array}{ll}\text { 16.2 The partnership agreement } & 123\end{array}$

$\begin{array}{ll}16.3 \text { Capital accounts } & 123\end{array}$

$\begin{array}{ll}16.4 \text { Partners' current accounts } & 124\end{array}$

$\begin{array}{ll}16.5 \text { Partners' loan accounts } & 124\end{array}$

$\begin{array}{ll}\text { 16.6 Profit and loss appropriation account } & 124\end{array}$

$\begin{array}{ll}16.7 \text { Worked examples } & 124\end{array}$

$\begin{array}{ll}16.8 \text { Further exercises } & 129\end{array}$

17 Partnership Accounts: 2

$\begin{array}{lll}17.1 & \text { Introduction } & 136\end{array}$

$\begin{array}{ll}17.2 \text { Goodwill } & 136\end{array}$

$\begin{array}{ll}17.3 \text { Goodwill on amalgamation of businesses } & 137\end{array}$

$\begin{array}{ll}17.4 \text { Goodwill on admission of new partner } & 137\end{array}$

$\begin{array}{ll}17.5 \text { Writing off goodwill } & 137\end{array}$

$\begin{array}{ll}\text { 17.6 Worked examples } & 138\end{array}$

$\begin{array}{lll}17.7 & \text { Further exercises } & 141\end{array}$

18 Manufacturing Accounts $\quad 145$

$\begin{array}{ll}18.1 \text { Introduction } & 145\end{array}$

$\begin{array}{ll}18.2 \text { Types of cost } & 145\end{array}$ 
18.3 Opening and closing stocks 145

$\begin{array}{ll}18.4 \text { Transfer price of finished goods produced } & 146\end{array}$

$\begin{array}{ll}18.5 \text { Manufacturing accounts for separate products } & 147\end{array}$

$\begin{array}{ll}18.6 \text { Fixed and variable costs } & 147\end{array}$

$\begin{array}{lll}18.7 \text { Worked examples } & 147\end{array}$

18.8 Further exercises 151

19 Stock Valuation $r$

$\begin{array}{lll}19.1 \text { Introduction } & 157\end{array}$

$\begin{array}{ll}19.2 \text { Bases of stock valuation } & 157\end{array}$

$\begin{array}{rr}19.3 \text { Valuation of stocks at cost } & 158\end{array}$

$\begin{array}{ll}19.4 \text { Choice of method } & 159\end{array}$

$\begin{array}{lll}19.5 \text { Effect on profits } & 159\end{array}$

$\begin{array}{ll}19.6 \text { Worked examples } & 160\end{array}$

$\begin{array}{lll}19.7 & \text { Further exercises } & 161\end{array}$

20 Comprehension and Interpretation of Accounts 165

20.1 Introduction 165

$\begin{array}{ll}20.2 \text { Return on capital employed (ROCE) } & 165\end{array}$

20.3 Profitability ratios 165

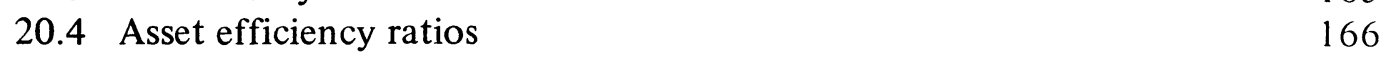

$\begin{array}{ll}20.5 \text { Liquidity ratios } & 167\end{array}$

$\begin{array}{ll}20.6 \text { Worked examples } & 168\end{array}$

$\begin{array}{lll}20.7 & \text { Further exercises } & 171\end{array}$

21 Accounts of Limited Companies $\quad 174$

$\begin{array}{ll}21.1 \text { Introduction } & 174\end{array}$

$\begin{array}{ll}21.2 \text { Share capital } & 174\end{array}$

$\begin{array}{lll}21.3 & \text { Debentures } & 175\end{array}$

$\begin{array}{ll}21.4 \text { Dividends } & 175\end{array}$

$\begin{array}{ll}21.5 \text { Retained profits } & 175\end{array}$

$\begin{array}{ll}21.6 \text { General reserve } & 175\end{array}$

$\begin{array}{ll}21.7 \text { Shareholders' funds } & 176\end{array}$

$\begin{array}{ll}21.8 \text { Profit and loss appropriation account } & 176\end{array}$

$\begin{array}{ll}21.9 \text { Worked examples } & 176\end{array}$

$\begin{array}{ll}21.10 \text { Further exercises } & 180\end{array}$

22 Forecasts and Budgets $\quad 184$

$\begin{array}{ll}22.1 & \text { Introduction } \\ 22.2 & 184\end{array}$

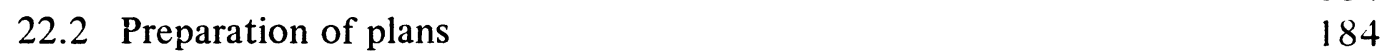

$\begin{array}{lll}22.3 \text { Worked examples } & 184\end{array}$

$\begin{array}{lll}22.4 & \text { Further exercises } & 187\end{array}$

$\begin{array}{ll}\text { Index } & 193\end{array}$ 


\section{Acknowledgements}

The University of London Entrance and School Examinations Council accepts no responsibility whatsoever for the accuracy or method in the answers given in this book to actual questions set by the London Board.

Acknowledgement is made to the Southern Universities' Joint Board for School Examinations for permission to use questions taken from their past papers but the Board is in no way responsible for answers that may be provided and they are solely the responsibility of the author.

The Associated Examining Board, the University of Oxford Delegacy of Local Examinations. and the Scottish Examination Board wish to point out that worked examples included in the text are entirely the responsibility of the author and have neither been provided nor approved by the Board.

The author and publishers wish to thank the following who have kindly given permission for the use of copyright material: The Associated Examining Board, The Scottish Examination Board, The Southern Universities' Joint Board, The University of London School Examinations Board, The University of Oxford Delegacy of Local Examinations for questions from past examination papers.

Every effort has been made to trace all the copyright holders, but if any have been inadvertently overlooked, the publishers will be pleased to make the necessary arrangement at the first opportunity. 